\title{
Local Layer Stacking and Structural Disorder in Graphene Oxide Studied via Scanning Electron Diffraction.
}

\author{
Alexander S. Eggeman ${ }^{1}$, Rowan K. Leary ${ }^{1}$, Duncan N. Johnstone ${ }^{1}$ and Paul A. Midgley ${ }^{1}$ \\ 1. Department of Materials Science and Metallurgy, University of Cambridge, Cambridge, UK
}

Graphene oxide (GO) is a structurally complex and variable material comprising sheets of carbon atoms functionalized with a variety of moieties, including carboxyl, epoxy and hydroxyl groups. Potential applications span optoelectronics, energy storage, environmental science and biotechnology. The structural complexity necessitates the application of versatile and information rich characterization techniques. Conventional and scanning transmission electron microscopy [(S)TEM] has provided numerous insights into GO structure [e.g. 1-3], but the detail attained has not reached those familiar from studies of more pristine graphene [4-6]. Here, scanning electron diffraction (SED) is applied to obtain more comprehensive insights into the local layer stacking and structural disorder in GO.

SED involves scanning the electron beam across the sample and recording an electron diffraction pattern at each probe position. Real and reciprocal space information is obtained with nanometer spatial resolution and rapid acquisition enables potentially beam sensitive materials, such as GO, to be studied. The 4D-SED dataset contains a wealth of information that can be extracted computationally post-facto [7]. In the case of GO, interpretation of the electron diffraction requires consideration of the effect of disorder, particularly on the diffracted intensities. These effects were studied via simulation, as shown in Figure 1a. Importantly, the metric commonly used to determine layer number in graphene was found to become unreliable. In graphene, monolayer material has first order reflections stronger than second order reflections with this ratio being reversed in multilayer material, but this breaks down for typical levels of disorder in GO. Taking account of the effects of disorder a region of GO film was analyzed (Figure 1b) revealing mono-, bi- and tri-layer areas (Figure 1c and d) consistent with the simulations.

Images formed by plotting the intensity within a subset of pixels containing particular reflections in the diffraction plane as a function of probe position revealed contrasts of light/dark fringes or 'striations' across the field of view, shown in Figure 2. This contrast is attributed to local displacements between the graphitic sheets. When the layers are shifted away from the ideal AB sequence, the $(1,0)$-type reflections show a significant variation in intensity. We interpret the VDF intensity striation effect here as the misregistry between buckled graphitic sheets or between a buckled GO monolayer and a flat GO bilayer, where the buckling introduces displacement similar to a set of partial dislocations. Close inspection of the average diffraction from the region (Figure 2a) showed that as well as the tri-layer material producing striation images, two GO monolayers with distinct rotational orientations are detectable. Orientation variations in the GO tri-layer were mapped by comparing the experimental diffraction to simulated patterns in a template library. Two regions with rotation about the graphitic c-axis by $>2^{\circ}$ were identified (Figure 2c). One of these regions has an abrupt linear boundary with the majority of the film, which is identified as a topographical feature such as a kink or fold in one of the layers. The other rotated region has a curved boundary that is otherwise coherent making this most likely a low-angle grain boundary. 
[1] Wilson, N. R. et al. ACS Nano 3, (2009) p. 2547-2556.

[2] Dave, S. H. et al. ACS Nano 10 (2016), p. 7515-7522.

[3] Mkhoyan, K. A. et al. Nano Letters 9, (2008), 1058-1063.

[4] Huang, P. et al, Nature 469 (2011), p. 389-392.

[5] Butz, B. et al, Nature 505 (2014), p.533-537.

[6] Meyer, J. C. et al, Solid State Communications 143 (2007), p. 101-109.

[7] Moeck, P. et al, Cryst. Res. Technol. 46 (2011), p. 589-606.

[8] This work has received funding from the EU Seventh Framework Programme under Grant Agreement 312483 -

ESTEEM2 and from the ERC Ref. 291522 3DIMAGE. RKL acknowledges a Junior Research Fellowship at Clare College. ASE acknowledges funding from the Royal Society.
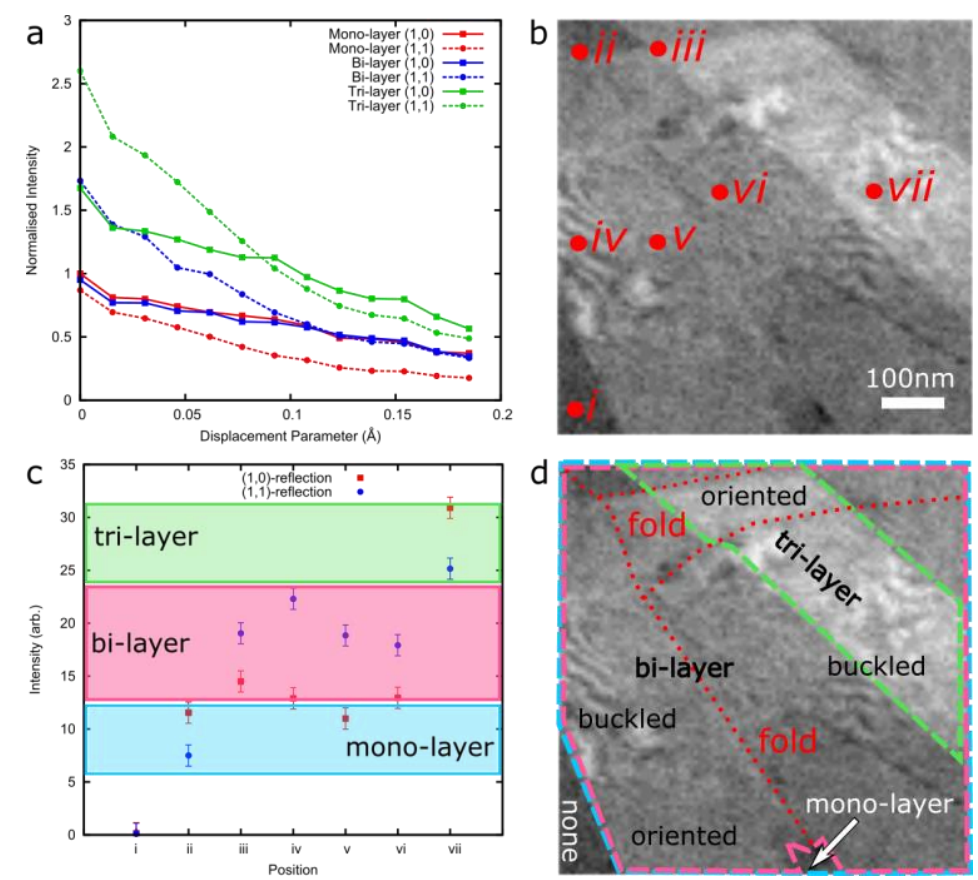

Figure 1. Analysis of multi-layer GO. (a) Simulated intensities of $1^{\text {st }}$ and $2^{\text {nd }}$ order reflection intensities as a function of disorder parameter. (b) A DVF image with eight regions of interest labelled the absolute reflection intensities in these regions are plotted in (c). (d) the VDF image with different GO layers marked out as well as defects and mis-registry labelled.
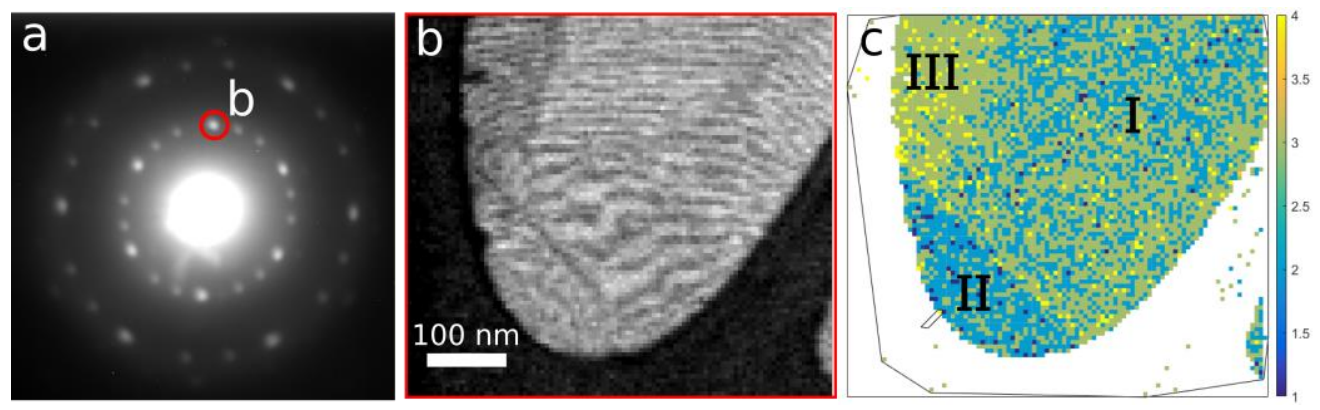

Figure 2. SED analysis of a continuous GO film. (a) summed diffraction from the entire scan, the labelled reflection was used to produce the VDF image in (b). c) Orientation map produced from the experimental diffraction data showing the clockwise rotation of the graphitic lattice about the c-axis. 